\title{
Safety and immunogenicity of an oral tablet norovirus vaccine, a phase I randomized, placebo-controlled trial
}

\author{
Leesun Kim, ${ }^{1}$ David Liebowitz, ${ }^{1}$ Karen Lin, ${ }^{1}$ Kassandra Kasparek, ${ }^{1}$ Marcela F. Pasetti, ${ }^{2}$ Shaily J. Garg, \\ Keith Gottlieb, ${ }^{1}$ George Trager, ${ }^{1}$ and Sean N. Tucker ${ }^{1}$ \\ 'Vaxart, South San Francisco, California, USA. ²enter for Vaccine Development, University of Maryland School of Medicine, \\ Baltimore, Maryland, USA.
}

\begin{abstract}
BACKGROUND. Noroviruses are the leading cause of epidemic acute gastroenteritis and foodborne diarrheal disease in humans. However, there are no approved vaccines for noroviruses. Potential correlates of protection identified through human challenge studies include mucosal IgA, memory $B$ cells, and serum-blocking antibody titers (BT50).
\end{abstract}

METHODS. We conducted a single-site, randomized, double-blind, placebo-controlled clinical trial of an oral norovirus vaccine to determine safety and immunogenicity. This tablet vaccine is comprised of a nonreplicating adenovirus-based vector expressing the VP1 gene from the GI.1 norovirus strain and a double-stranded RNA adjuvant. Sixty-six adult subjects meeting inclusion/ exclusion criteria were randomized 2:1 to receive a single vaccine dose or placebo, respectively. Immunogenicity was primarily assessed by serum BT50. Additional outcomes included serum ELISA titers, fecal and saliva antibody titers, memory and antibody-secreting cell (ASC) frequency, and $B$ cell phenotyping.

RESULTS. The vaccine was well-tolerated, with no dose-limiting toxicities. Adverse events were mild or moderate. The primary immunological endpoint (increase in BT50 titers) was met in the high-dose group $(P=0.0003$ ), with $78 \%$ showing a $\geq 2$-fold rise in titers after a single immunization. Vaccine recipients also developed mucosally primed VP1-specific circulating ASCs, IgA+ memory $B$ cells expressing gut-homing receptor ( $\alpha 4 \beta 7)$, and fecal IgA, indicating substantial and local responses potentially relevant to prevent norovirus infection.

Role of funding source: The sponsor of the study (Vaxart) designed the protocol and had overall responsibility for its conduct. All authors vouch for the accuracy and completeness of the data. L. Kim, D. Liebowitz, and S.N. Tucker had full access to all the data in the study and had final responsibility for the decision to submit for publication.

Conflict of interest: L. Kim, K. Lin, K. Kasparek, K. Cottlieb, D. Liebowitz, G. Trager, S.J. Garg, and S.N. Tucker are employees of Vaxart and have received stock options and compensation as part of their employment.

\section{Submitted: March 15, 2018 \\ Accepted: May 25, 2018 \\ Published: July 12, 2018}

\section{Reference information:}

JCI Insight. 2018;3(13):e121077.

https://doi.org/10.1172/jici.

insight.121077.
CONCLUSION. This oral norovirus vaccine was well-tolerated and generated substantial immune responses, including systemic and mucosal antibodies as well as memory IgA/IgC. These results are a major step forward for the development of a safe and immunogenic oral norovirus vaccine.

TRIAL REGISTRATION. ClinicalTrials.gov NCT02868073.

FUNDING. Vaxart.

\section{Introduction}

Noroviruses are the leading cause of epidemics of acute gastroenteritis and foodborne disease worldwide $(1,2)$. Infection is classically characterized by severe vomiting, diarrhea, and abdominal cramping for 28-60 hours within 10-51 hours of exposure (3). The virus is transmitted by the fecal/oral route, and because of the durability of the virus particles on exposed surfaces (4), severe outbreaks can occur in tight, close-quartered conditions, such as hospitals, military barracks, schools, camps, and ships (5-7).

There are currently no licensed vaccines for norovirus, and lack of an adequate animal model to test efficacy has hindered vaccine development. The most advanced vaccine candidates to date have relied on cell culture-based expression of norovirus VP1, which spontaneously forms a virus-like particle (VLP) that can be subsequently purified. Purified VLPs have been given orally, intranasally, and intramuscularly to mice and humans, usually with adjuvants that improve immunogenicity (reviewed by Riddle et al.) (8). Vaccine approaches in humans have focused on the main disease-causing genogroups of norovirus, GI 
and GII, using VLP preparations. In a human clinical trial, intranasal administration of a VLP (from the GI.1 Norwalk strain or genotype) plus monophosphoryl lipid A and chitosan, reduced norovirus-associated acute gastroenteritis following homologous challenge (9). A subsequent human study tested an intramuscular bivalent vaccine containing components from two different genogroups (VLP from GI.1 and a consensus $V L P$ sequence derived from 3 GII.4 norovirus strains) (10). This vaccine was well tolerated and immunogenic, and it decreased the severity of illness after challenge with GII.4 norovirus. However, the incidence of GII.4 norovirus-associated acute gastroenteritis was not significantly reduced (10).

Norovirus strains are genetically diverse, and infection with a single strain does not confer long-term sterilizing immunity but rather short-term protection (11). Similarly, long-term immunity has been difficult to achieve for some enteric vaccines, possibly due to the rapid decline of intestinal IgA compared with longer-term serum IgG responses (12). Mucosal IgA likely plays a pivotal role in norovirus protection, but human challenge studies have shown that serum IgA, memory B cell responses, and serum histo-blood group antigen-blocking (HBGA-blocking) titers (BT50) are all potential immunological correlates of protection (9, 13-15). Vaccine development must overcome these challenges and determine true immunological correlates of efficacy. An easy to administer vaccine capable of generating a broader immune response through activation of multiple lines of defense could provide a solution to the challenges of norovirus infection.

Vaxart is developing an oral vaccine platform, which has been tested successfully in multiple phase I human studies with an $\mathrm{H} 1$ influenza vaccine candidate. The orally administered vaccine tablet platform is well tolerated and generates robust neutralizing antibody responses to influenza as well as mucosal immune responses $(16,17)$. In poliovirus human vaccine studies, Dey et al. have shown that a virus-specific antibody-secreting cell (ASC) response can be used as a measure of mucosal immunity induction following oral vaccination against an enteric pathogen (18). In their study, virus-specific ASCs that expressed the $\alpha 4 \beta 7$ integrin were used as a biomarker for mucosal immunity, since $\alpha 4 \beta 7$ is involved in selective B cell homing to mucosal intestinal tissues. The Vaxart vector approach has also been shown to induce IgA ASCs and $\alpha 4 \beta 7$-expressing B cells (16) in humans, suggesting that this vaccine platform is capable of initiating an intestinal immune response that could be especially advantageous for deterring a pathogen that infects the intestinal mucosa.

Vaxart has now constructed a gene-based vaccine aimed at inducing protective immunity and preventing norovirus illness using the same platform investigated in the influenza trials. This vaccine is expected to express VP1 in vivo in human intestinal epithelial cells, in a manner similar to natural norovirus infection. Additionally, this gene-based vaccine approach allows for multiple antigens to be expressed from the same vector and potentially facilitate rapid changes to the vaccine antigen as new norovirus strains evolve. These technological advantages could prove useful in deterring a genetically diverse virus, such as norovirus. Here, we present and discuss the safety and immunogenicity profile of this oral tableted norovirus vaccine candidate in a first-in-human clinical study.

\section{Results}

\section{Demographics}

Between July and September of 2016 in Nebraska, USA, 152 subjects were screened and 66 subjects were enrolled and dosed. Sixty-five of sixty-six subjects completed safety and immunogenicity assessments through the active phase (day 28), and one subject in the placebo group withdrew before day 7 (Figure 1). Subject demographics are provided in Table 1.

\section{Summary of adverse events}

All solicited adverse events (AEs) reported in this trial $(n=46)$ were grade 1 or 2 in severity, with the majority being mild events (44 grade 1 and 2 grade 2 events). The percentage of subjects with any solicited symptoms was similar between test and placebo treatments (Table 2). In the first 7 days following study drug administration, 35 study subjects had at least one solicited AE reported, with 25 of 46 (54\%) subjects in the VXAG1.1-NN vaccine groups and 10 of $20(50 \%)$ subjects in the placebo group (Table 2). Diarrhea and headache were the most common solicited symptoms following VXA-G1.1-NN administration, both of which were reported by 15 of $23(33 \%)$ subjects in the treated groups. Headache and nausea were reported evenly across treatments, including placebo. The only solicited symptom demonstrating a statistically significant difference from placebo was diarrhea $(P=0.0275)$, which was reported by 11 subjects in the high-dose group. Nine of the eleven subjects reported mild severity diarrhea, while two subjects reported moderate severity episodes 


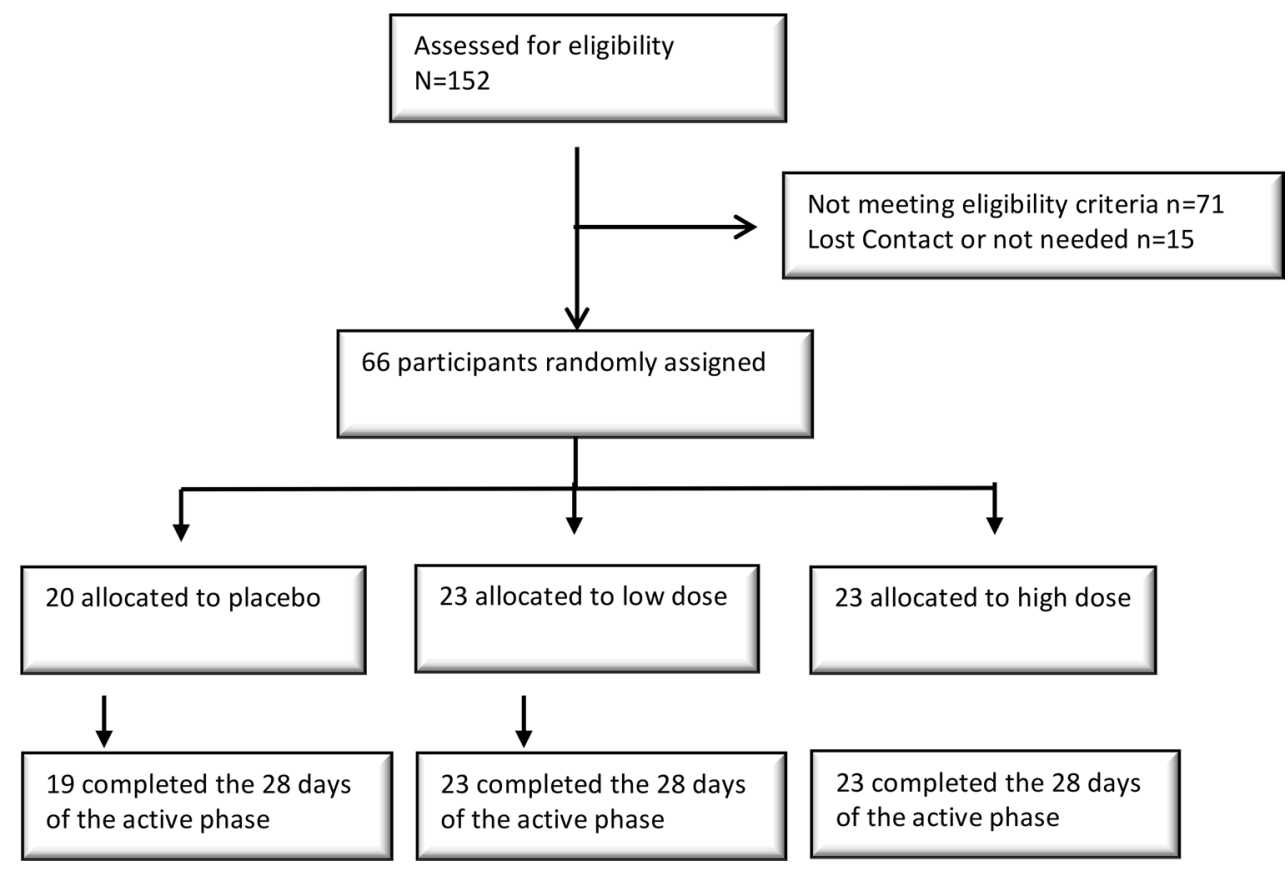

Figure 1. Trial profile, including enrollment.

following the high-dose vaccine. Onset of diarrhea (verbatim term "loose stools") ranged from day 1 to day 6 following vaccine administration, and most episodes resolved within 1 day. Because there were no reports of increased frequency of diarrhea in other clinical studies using the same platform with a different transgene antigen (17), we hypothesized that VP1 expression in the intestine could be causing the reported diarrhea. However, a subsequent norovirus vaccine clinical study in which subjects were given two high doses of vaccine from the same good manufacturing practice (GMP) lot (ClinicalTrials.gov NCT03125473) 28 days apart showed no such propensity for loose stools; only 1 of 15 subjects (6.7\%) reported diarrhea (D. Liebowitz, unpublished observations). These results suggest that the diarrhea finding is a statistical aberration. At no point did any loose stool symptoms effect normal activity, such as work or school, and none of them required treatment with antidiarrheal medications or rehydration therapy.

A total of 83 unsolicited treatment emergent AEs (TEAEs) were reported by 33 of the 66 subjects within the first 28 days after dosing, with slightly more placebo subjects (12 of 20,60\%) reporting AEs than lowdose (11 of $23,48 \%$ ) or high-dose vaccinated subjects (10 of $23,44 \%$ ). Headache was the most common

Table 1. Baseline demographics

\begin{tabular}{|c|c|c|c|c|}
\hline & Placebo $(n=20)$ & Low dose $(n=23)$ & High dose $(n=23)$ & Total $(n=66)$ \\
\hline Male & $9(45 \%)$ & $10(43 \%)$ & $13(57 \%)$ & $32(48 \%)$ \\
\hline Asian & $1(5 \%)$ & $1(4 \%)$ & $0(0 \%)$ & $2(3 \%)$ \\
\hline Black or African American & $1(5 \%)$ & $1(4 \%)$ & $4(17 \%)$ & $6(9 \%)$ \\
\hline Asian or Pacific Islander & $1(5 \%)$ & $0(0 \%)$ & $0(0 \%)$ & $1(2 \%)$ \\
\hline Hispanic or Latino & $4(20 \%)$ & $2(9 \%)$ & $0(0 \%)$ & $6(9 \%)$ \\
\hline Not Hispanic or Latino & $16(80 \%)$ & 21 (91\%) & $23(100 \%)$ & 60 (91\%) \\
\hline Body mass index & $27.4(4.4)$ & $26.5(3.8)$ & $29.2(3.4)$ & $27.7(4.0)$ \\
\hline
\end{tabular}


Table 2. Solicited adverse events for vaccine and placebo groups

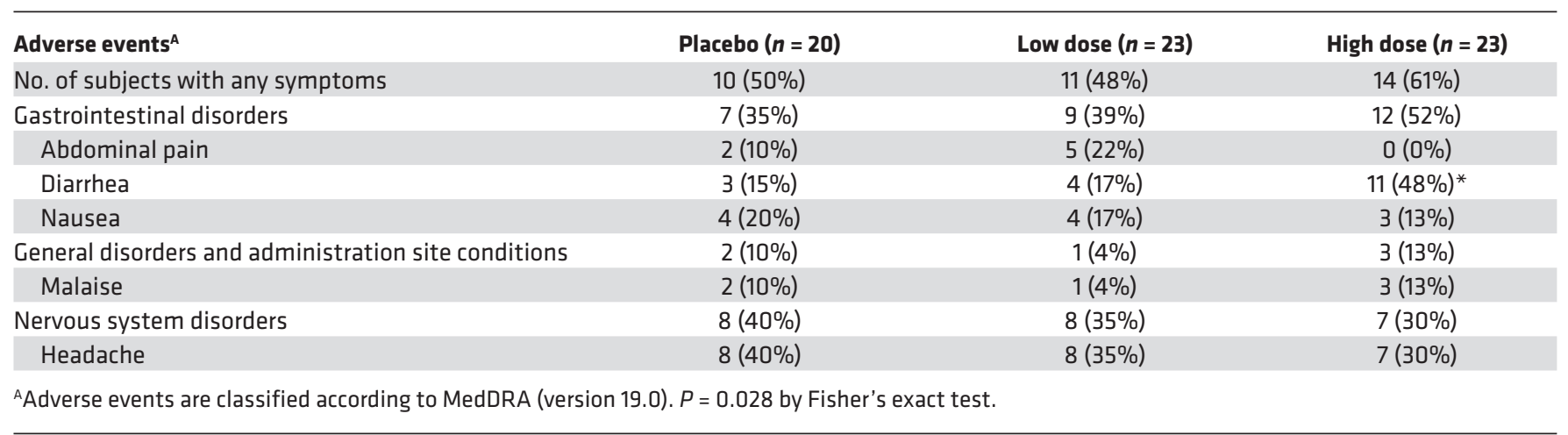

AE reported in all treatments. Most TEAEs were mild or moderate in severity. The principal investigator (PI) considered 28 TEAEs possibly related, 42 unlikely related, and 13 not related. A summary of unsolicited TEAEs, by number of subjects reporting at least one event, is presented within the Supplemental Table 1 (supplemental material available online with this article; https://doi.org/10.1172/jci.insight.121077DS1).

\section{Immunogenicity results}

Primary immunogenicity analysis. Antibody responses to norovirus VP1 were assessed by BT50 assay. Titers were measured by using either Lewis B $\left(\mathrm{Le}^{\mathrm{b}}\right)$ or type $\left.\mathrm{H} 1 \mathrm{H} 1\right)$ synthetic glycan as the coating antigen. Using the Le $\mathrm{L}^{\mathrm{b}}$ assay, 14 of 23 (61\%) subjects had a 2-fold rise in the low-dose group, and 18 of 23 (78\%) had a 2 -fold rise or greater in the high-dose group. One subject in the placebo group had a greater than 2-fold rise. On day 28 , the geometric mean titer (GMT), as determined by the $\mathrm{Le}^{\mathrm{b}}$ assay, for the low-dose vaccine group was 59.0 (95\% CI 33.0-105.4), a 2.3-fold geometric mean fold rise (GMFR) over the initial GMT of 26.2 (95\% CI 16.6-41.2) at baseline. The GMT for the high-dose vaccine group was 98.5 (95\% CI 64.4-150.7), a 3.8-fold GMFR over the initial GMT of 25.8 (95\% CI 18.3-36.2) at baseline. Similar observations were made for titers measured with $\mathrm{H} 1$. The high-dose group had significantly increased titers compared with placebo on day 28 , as determined by either $\operatorname{Le}^{\mathrm{b}}(P=0.0003)$ or $\mathrm{H} 1(P=0.001)$ BT50 assay (Table 3$)$. The effects of blood type and preexisting immunity to adenovirus serotype 5 (Ad5) were insignificant in terms of fold rise in BT50 titers (Supplemental Figures 1 and 2).

Serum antibody response. Serum antibody responses to the vaccine were measured before and after immunization by IgG and IgA ELISA. The changes in titers at the median effective concentration $\left(\mathrm{EC}_{50}\right)$ between days 0 and 28 were calculated for each subject. The titers in the vaccine groups were significantly $(P<0.0001)$ increased, and the majority of those subjects had an increase in antibody responses after immunization. The average changes for $\operatorname{IgA}$ and $\operatorname{IgG~} \mathrm{EC}_{50}$ for the low-dose group were 16-fold and 7.1-fold, respectively. Similar-

Table 3. GMT and statistical significance for Le ${ }^{b}$ and H1 BT50 assays

\begin{tabular}{|c|c|c|c|c|c|c|c|c|}
\hline $\begin{array}{l}\text { HBGAs }^{A} \\
\text { Group }\end{array}$ & $\begin{array}{l}\text { Day 0 CMT } \\
(95 \% \mathrm{CI})\end{array}$ & $\begin{array}{c}\text { Day } 28 \text { CMT } \\
(95 \% \mathrm{CI})\end{array}$ & GMFR & $P$ value $^{B}$ & $\begin{array}{l}\text { Day 0 GMT } \\
(95 \% \mathrm{Cl})\end{array}$ & $\begin{array}{c}\text { Day } 28 \text { CMT } \\
(95 \% \mathrm{CI})\end{array}$ & GMFR & $P$ value $^{2}$ \\
\hline $\begin{array}{l}\text { Low dose } \\
(n=20)\end{array}$ & $\begin{array}{c}26.2 \\
(16.6-41.2)\end{array}$ & $\begin{array}{c}59.0 \\
(33.0-105.4)\end{array}$ & 2.3 & 0.0459 & $\begin{array}{c}22.8 \\
(15.6-33.5)\end{array}$ & $\begin{array}{c}50.0 \\
(29.1-85.8)\end{array}$ & 2.2 & 0.045 \\
\hline $\begin{array}{l}\text { High dose } \\
(n=23)\end{array}$ & $\begin{array}{c}25.8 \\
(18.3-36.2)\end{array}$ & $\begin{array}{c}98.5 \\
(64.4-150.7)\end{array}$ & 3.8 & 0.0003 & $\begin{array}{c}22.5 \\
(16.6-30.5)\end{array}$ & $\begin{array}{c}69.7 \\
(47.2-102.9)\end{array}$ & 3.1 & 0.0013 \\
\hline \multicolumn{4}{|c|}{ Overall significance } & 0.0017 & \multicolumn{3}{|c|}{ Overall significance } & 0.0063 \\
\hline
\end{tabular}

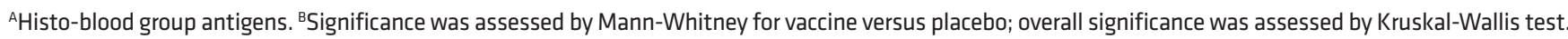


A

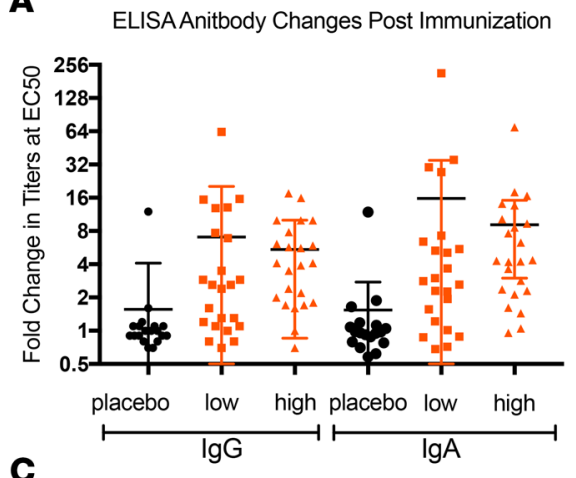

B
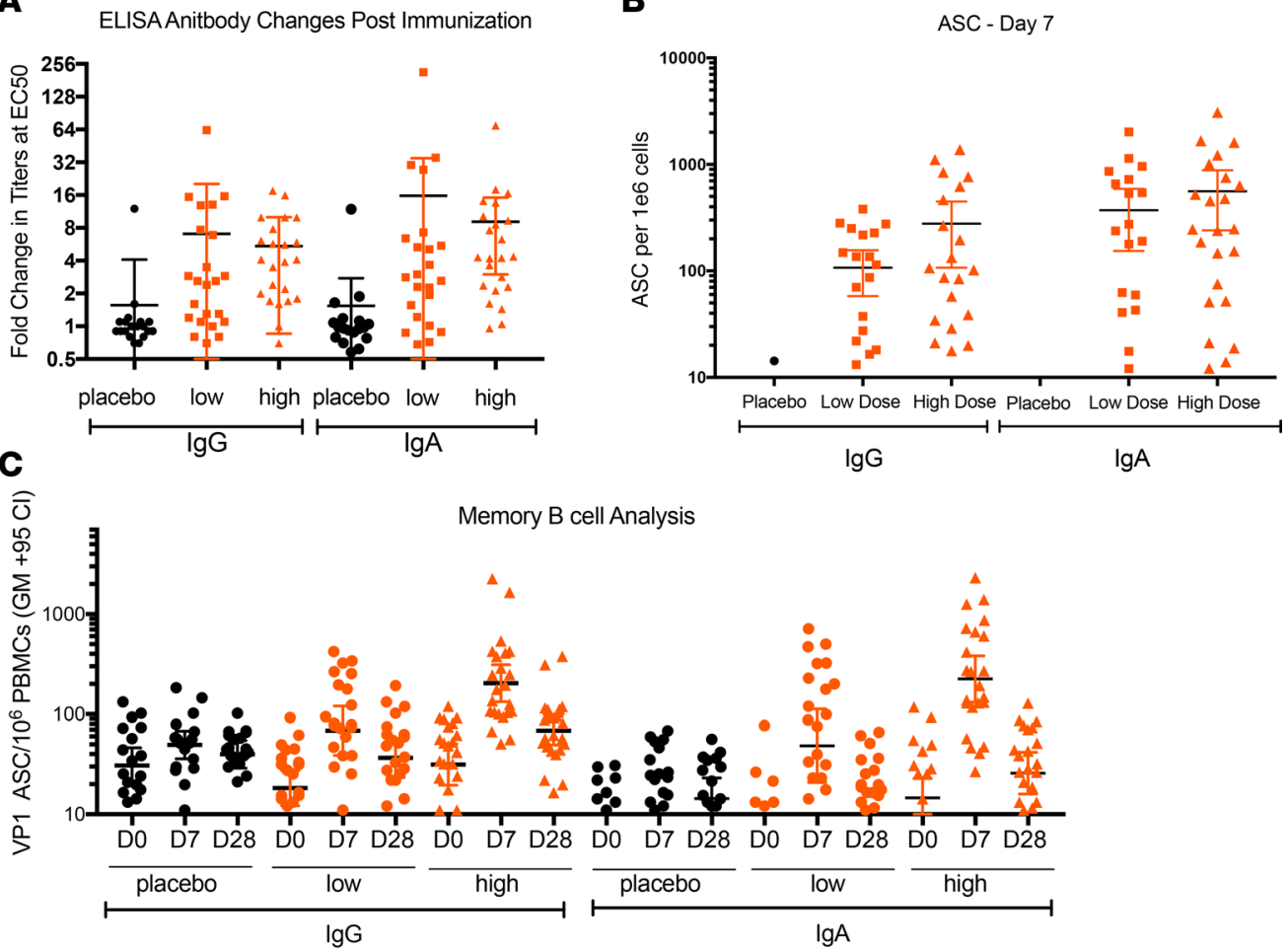

D

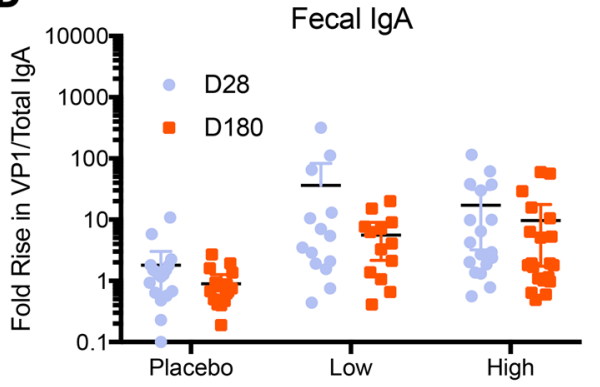

E

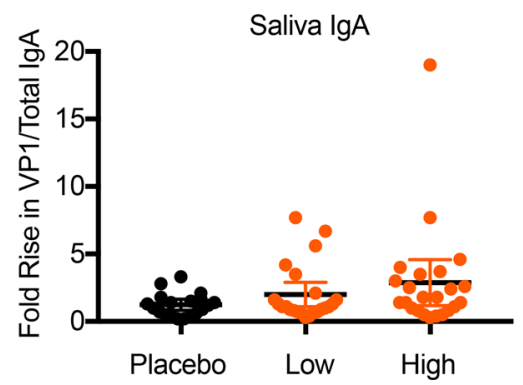

Figure 2. Immune responses to norovirus. Unless otherwise noted, horizontal bars represent average increase, with thinner lines indicating $95 \%$ confidence interval. $n=20$ for placebo group, $n=23$ for the vaccine groups. (A) IgA or IgG ELISA titers after immunization. Data represent fold increases in antibody levels 28 days after vaccination (compared with day 0 ) for all subjects divided by treatment group (each symbol represents an individual subject). Significance was assessed by Kruskal-Wallis. (B) Norovirus VP1-specific IgC and IgA ASC counts on day 7 after vaccination. Significance was assessed by Kruskal-Wallis. (C) Norovirus VP1-specific memory B cell counts. Longer black horizontal lines represent the geometric mean, and error bars indicate the $95 \%$ confidence interval. Significance was assessed by Kruskal-Wallis. (D) Norovirus VP-specific fecal responses. Samples were removed when total IgA was below the detection limit. Sample size of each group are as follows: high dose (day 0-28), $n=$ 19; high dose (day 0-180), $n=21$; low dose (day 0-28), $n=20$; low dose (day 0-180), $n=16$; placebo (day $0-28$ ), $n=18$; placebo (day 0-180), $n=16$. Significance was assessed by Mann-Whitney or Fisher's exact test. (E) Norovirus VP-specific saliva IgA responses. In $\mathbf{D}$ and $\mathbf{E}$, data represent fold increase in specific IgA/ total IgA for each group with each time pair point plotted.

ly, the average changes for IgA and IgG in the $\mathrm{EC}_{50}$ for the high-dose group were 9-fold and 5.4-fold, respectively. The changes in $\mathrm{EC}_{50}$ are plotted for each subject, separated by group (Figure 2A).

Memory and ASC responses. The ability of the vaccine to induce antigen-specific antibody-producing $\mathrm{B}$ cells in peripheral blood was measured by ASC assay (Figure 2B). In the low-dose group, 16 of $23(70 \%)$ subjects had positive IgA and IgG ASC responses 7 days after vaccination, and in the high-dose group, 19 of 23 (83\%) subjects had these positive responses. Background ASCs were generally negligible on day 0 . For the high-dose group, averages of 561 IgA ASCs (95\% CI 240.5-881.3) and 278 IgG ASCs (95\% CI 107.3-448.9), each per $1 \times 10^{6}$ peripheral blood mononuclear cells (PBMCs), were detected on day 7. For the low-dose group, averages of 372 IgA ASCs (95\% CI 154.1-589.9) and 107 IgG ASCs (95\% CI 58.1-156.6) were found on day 7. The placebo group had no significant response, with an average of 3.3 IgA ASC spots (95\% CI 2.0-4.5) and 2.2 IgG ASC spots (95\% CI 0.52-3.88), each per $1 \times 10^{6} \mathrm{PBMCs}$, on day 7. The vaccine treated groups were significantly different than the placebo group in terms of their ability to elicit an IgG or an IgA ASC response at day $7(P<0.0001$ by Kruskal-Wallis, $P<0.0001$ by Mann-Whitney). There was no statistical difference in the number of spots for IgA and IgG ASCs between the high- and low-dose groups ( $P=0.21$ for IgA and $P=0.28$ for IgG by Mann-Whitney).

Expansion of antigen-specific memory B cells after immunization was investigated after culturing PBMCs with polyclonal stimulators $(19,20)$. The numbers of VP1-specific IgG memory B cells were higher than those of IgA memory B cells in the day 0 samples (Figure 2C). After immunization, the response at day 7 was higher for IgA memory B cells. In the high-dose group, the GMFR for IgA was 15.3, while IgG 
A

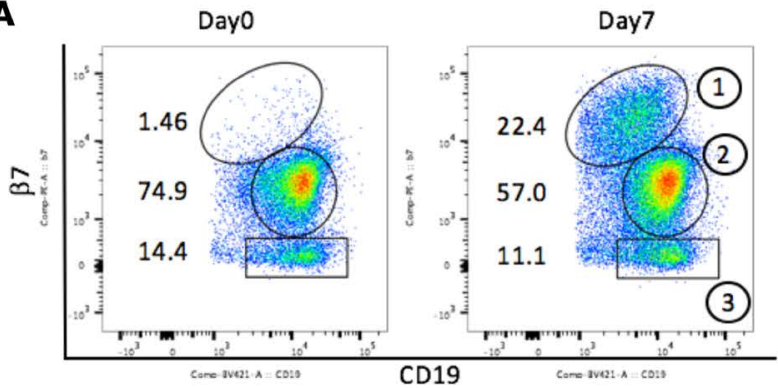

B

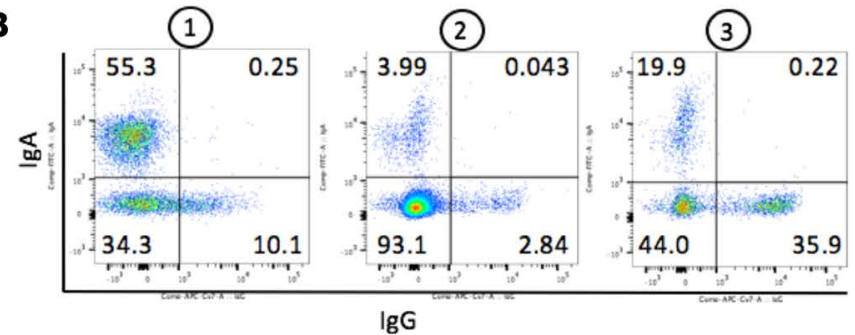

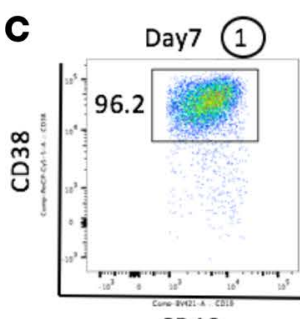

CD19

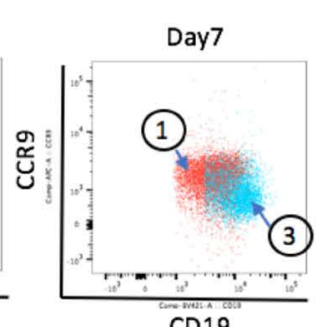

CD19
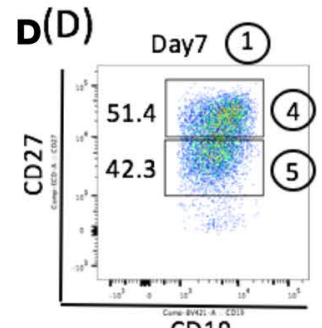

CD19
$\mathbf{E}$

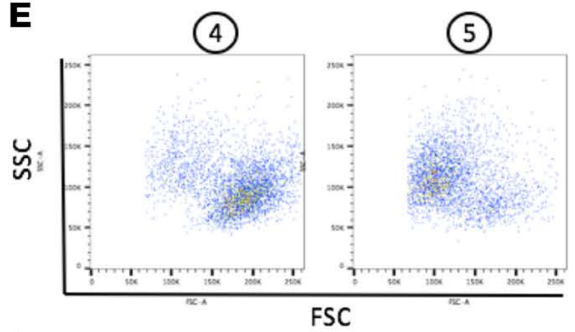

$\mathbf{F}$
(4)

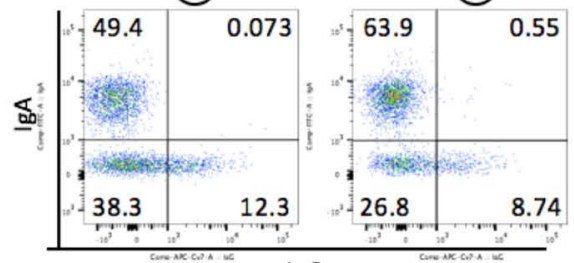

IgG

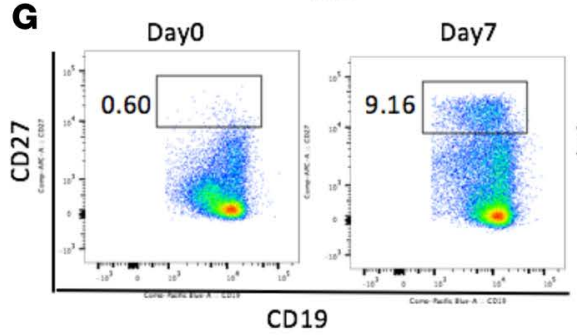

Day7
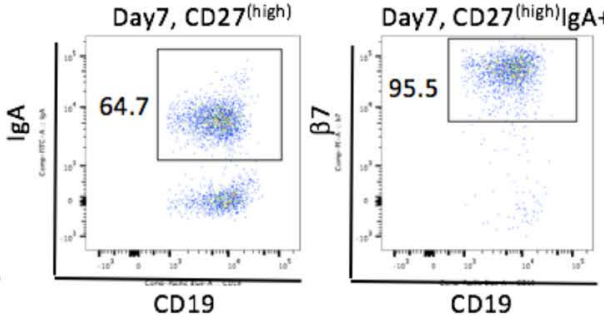

Figure 3. Generation of intestinal homing plasmablasts and memory $B$ cells after vaccination. (A) A population of $\beta 7^{\text {hi }} B$ cells was detected 7 days after vaccination. Note that CD19 expression is slightly downregulated in $\beta 7^{\text {hi }}$ $B$ cells. Based on the levels of $\beta 7,3$ populations were present on day 7 B cells: $\beta 7^{\text {hi }}$ (noted as 1 ), $\beta 7^{\text {intermediate }}$ (noted as 2 ), and $\beta 7^{-}$(noted as 3 ). (B) Expression of IgA and IgG in $\beta 7^{\text {hi }}$ (noted as 1 ),

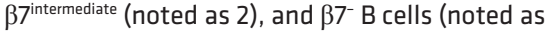
3). Higher frequencies of IgA-expressing $B$ cells were present in the $\beta 7^{\text {hi }}$ population than in other populations. (C) $\beta 7^{\text {hi }} B$ cells (noted as 1 ) coexpress high levels of the activation marker CD38 and CCR9 (red). The light blue color represents CCR9 expression on $\beta 7^{-} B$ cells (noted as 3 ).

(D) $\beta 7^{\text {hi }} B$ cells (noted as 1 ) contain both CD27 $7^{\text {hi }}$ plasmablasts and CD27intermediate memory $B$ cells. Populations 4 and 5 are defined by CD27 expression level. (E) FSC and SSC plots gated from populations 4 and 5. (F) Surface expression of IgA and IgC from populations 4 and 5. (C) The gating strategy for the graph in $\mathbf{H}$ : CD27 ${ }^{\text {hi }} B$ cells appeared on day $7 ; \lg A^{+} B$ cells were gated from CD27 $7^{\text {hi }} B$ cells; and then $\beta 7^{\text {hi }} B$ cells were gated from CD27 ${ }^{\text {hil }} g A^{+} B$ cells. (H) The percentage of $\beta 7^{\text {hi }}$ cells gated from $C D 27^{\text {hil }} g A^{+} B$ cells in day 7 PBMCs. Subjects from low-dose $\left(1 \times 10^{10}\right)$ and high-dose $\left(1 \times 10^{11}\right)$ groups were examined. The black horizontal bars represent means, with error bars indicating the $95 \%$ confidence interval. For A-G, data are from one subject, but the analysis was performed on all 66 subjects, and the results are representative of the responding vaccine subjects $(>70 \%)$. $\mathbf{H}$ shows the distribution of $\beta 7$ on all vaccine-treated subjects.

H

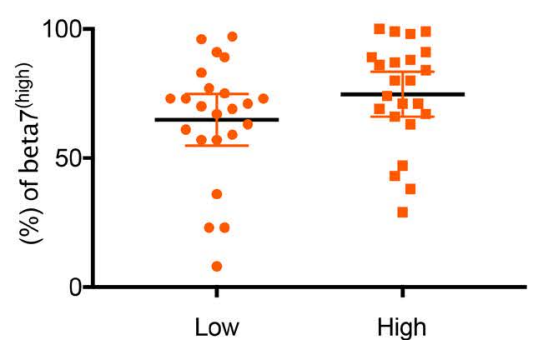


was 6.5 at day 7, before declining again at day 28. In the low-dose group, a GMFR of 7.4 for IgA and 3.7 for IgG was observed at day 7 , before it declined again at day 28. The decline from day 7 to day 28 may have resulted from homing of circulating B cells from the peripheral blood to the intestinal lymphoid tissues via expression of high levels of $\alpha 4 \beta 7$ (shown and discussed below). In the high-dose group at day 7, 20 of $23(87 \%)$ subjects and 19 of $23(83 \%)$ subjects had a $\geq 2$-fold increase for IgA and IgG, respectively, compared with day 0 values. In the low-dose group at day 7, 18 of $23(78 \%)$ subjects and 13 of $23(57 \%)$ subjects had $\mathrm{a} \geq 2$-fold increase for $\operatorname{IgA}$ and $\operatorname{IgG}$, respectively, compared with day 0 values. The vaccine subjects had significantly higher cell counts on day $7(P<0.0001$ by Kruskal-Wallis and $P<0.0001$ by Mann-Whitney), and the high dose subjects had significantly higher cell counts than low dose subjects $P=0.0038$ and $P=$ 0.0051 for IgG and IgA, respectively, when comparing low versus high groups by Mann-Whitney.

Mucosal antibody responses. Vaccine-induced VP1-specific mucosal IgA responses were evaluated in saliva and fecal extracts. Because the quantity of IgA can be highly variable in mucosal samples, the ratio of VP1-specific IgA to total IgA was determined for each sample; those with IgA levels below the detection limit were excluded from analysis. The increase in the ratio of specific IgA to total $\operatorname{IgA}$ was measured between baseline and day 28 (and baseline and day 180 for fecal IgA). In the high-dose group, 9 of 19 (47\%) subjects had 4-fold or greater IgA responses at day 28, and 9 of 21 (43\%) subjects had 4-fold or greater IgA responses at day 180 (Figure 2D); the average fold increases in the specific IgA to total IgA ratio were 17.2 and 9.7, respectively. Responses in the high-dose vaccine group were significantly higher than those in the placebo group, where 2 of $18(11 \%)$ subjects at day $28(P=0.029)$ and 0 of $16(0 \%)$ subjects at day $180(P=0.0049)$ were found to have 4-fold or greater increases after vaccination, with average increases of 1.8 and 1.0 (Figure 2D). Fecal IgA responses in the low-dose group were similar to those of the high-dose group, with 7 of 20 (35\%) subjects and 5 of 16 (31\%) subjects showing 4-fold increases on days 28 and 180, respectively. There was a trend of higher rates of response compared with the placebo group on day 28 , but the difference was only statistically significant on day $180(P=0.13, P=0.043)$. The average fold increase in specific IgA to total IgA ratio for the low-dose group was 36.2 on day 28 and 5.6 on day 180 (Figure 2D). Fewer saliva samples from vaccine recipients showed increases in the specific IgA to total IgA ratios between baseline and day 28 (Figure 2E). The high-dose and low-dose groups each had 4 subjects with a 4 -fold rise versus none for the placebo group $(P=0.11)$. The average fold increase in the specific IgA to total IgA ratio was 2.0 for the low-dose group, 2.9 for the high-dose group, and 1.2 for the placebo group.

Mucosally primed B cells expressing gut-homing markers. The trafficking and activation properties of B cells were assessed by examining the expression of the mucosal homing receptor $\alpha 4 \beta 7$ and the small intestinal chemokine CCR9 on circulating B cells. These markers have been associated with intestinal homing in humans (21). Previously, we reported that $\beta 7^{\mathrm{hi}} \mathrm{B}$ cells coexpressed $\alpha 4^{\mathrm{hi}}$ integrin and that a substantial number of the plasmablasts activated by oral immunization could be tracked by $\beta 7^{\text {hi }}$ expression. In the current study, a clear and distinct population of $\beta 7^{\text {hi }}$ B cells was observed after vaccination (Figure $3 A$ ). Up to $24 \%$ of B cells in the peripheral blood were found to express $\beta 7^{\text {hi }}$. The surface expression of $\operatorname{IgA}$ and $\operatorname{IgG}$ was then examined on $\beta 7^{\text {hi }} B$ cells to ascertain whether these orally primed $\beta 7^{\text {hi }} \mathrm{B}$ cells were committed for IgA production (enriched in surface $\operatorname{IgA}$ ). Representative results from one subject presented in Figure $3 \mathrm{~B}$ indicate that $55.3 \%$ of $\beta 7^{\text {hi }} \mathrm{B}$ cells (population 1) expressed surface IgA. In contrast to $\beta 7^{\text {hi }} \mathrm{B}$ cells, only $19.9 \%$ of $\beta 7^{-} \mathrm{B}$ cells (population 3 ) expressed surface IgA. The large double-negative IgG- and IgA-expressing $\beta 7^{\text {hi }} \mathrm{B}$ cells $(34.3 \%)$ in population 2 could be $\operatorname{IgM}$, $\operatorname{IgD}$, or even IgG-secreting B cells, because surface IgG expression is known to be downregulated on activated B cells (22). Further characterization of the $\beta 7^{\text {hi }} \mathrm{B}$ cell population 1 showed that they coexpressed high levels of CD38 (a marker of recent activation) and chemokine CCR9 (Figure 3C).

Antigen-specific ASCs and memory B cells were detected in peripheral blood, as shown in Figure 2, B and C. The mucosally primed expanded memory B cells and plasmablasts were phenotypically distinguished by flow cytometry through expression of CD27 on the $\beta 7^{\text {hi }} \mathrm{B}$ cells in each of these populations. Plasmablasts $\left(\mathrm{CD} 27^{\mathrm{hi}}\right.$, population 4 ) and memory $\beta 7^{\text {hi }} \mathrm{B}$ cells (CD27 $7^{\text {intermediate }}$, population 5) were elicited after oral immunization (Figure $3 \mathrm{D}$ ). Definition of these $\mathrm{CD} 27^{\mathrm{hi}}$ and intermediate gates was supported by analysis of cell size. As expected, $\mathrm{CD} 27^{\text {hi }}$ plasmablasts are larger in size (higher forward-side-scatter [FSC] signal) due to clonal expansion (Figure

$3 \mathrm{E}$ ). Both vaccine-induced plasmablast and memory B cell populations expressed surface IgA (Figure $3 \mathrm{~F}$ ).

We next investigated the expression of mucosal homing receptors on $\operatorname{IgA}^{+}$expressing plasmablasts to assess the pool of $\operatorname{IgA}^{+}$committed cells with capacity to return to the intestinal environment. The gating strategy is shown in Figure 3G. Selection of $\mathrm{CD} 27^{\text {hi }} \mathrm{B}$ cells was followed by gating $\operatorname{IgA}^{+} \mathrm{B}$ cells and then measuring the percentage of $\beta 7^{\text {hi }}$ cells. As seen in the day 0 plot (Figure 3G), very few CD2 $7^{\text {hi }} B$ cells were detected before vacci- 
nation $(0.60 \%)$. On day 7, the low-dose group mean percentage of $\mathrm{CD} 27^{\mathrm{hi}} \operatorname{IgA}^{+} \mathrm{B}$ cells that were $\beta 7^{\mathrm{hi}}$ was $64.83 \%$ (4.85 \pm SEM, 95\% CI 54.78-74.87), while the high-dose group mean percentage was $74.74 \%$ (4.21 \pm SEM, $95 \%$ CI 66.01-83.47) (Figure 3H). The difference between the two dose groups was not significant $(P=0.1298$ by unpaired $t$ test). These data indicate that the majority of $\operatorname{IgA}^{+}$plasmablasts acquired the intestinal homing receptor. The nonintestinal homing $\operatorname{IgA}^{+}$plasmablasts ( $\left.\beta 7^{\text {intermediate/- }}\right)$ could potentially traffic to other mucosal sites or to peripheral lymphoid tissue $(23,24)$. Taken together, these data suggest that the VXA-G1.1-NN tableted norovirus vaccine elicited robust intestinal homing IgA-expressing plasmablasts and memory B cells.

\section{Discussion}

Norovirus infection is a common affliction that spreads rapidly in confined locations and can lead to the closure of hospitals and camps and the quarantining of cruise and military ships. The societal economic costs due to norovirus infections have been estimated to be $\$ 60$ billion worldwide (25). The ability to provide long-term protection is difficult because of significant virus heterogeneity and a potential reliance on an intestinal IgA response, which has been known to be more transient than serum immune responses $(12,26)$. In this study, we found that an oral adenovirus vector-based vaccine approach was well tolerated and induced substantial effector and memory B cell mucosal immunity after a single administration. Fecal IgA responses were still elevated 6 months after immunization. In addition, there was no evidence that preexisting immunity to the recombinant Ad5 (rAd5) vector affected the ability to elicit a norovirus-specific immune response (see Supplemental Methods), which has been demonstrated in additional indications and studies using the same oral vaccine platform $(17,27)$.

Several VLP norovirus vaccines have been tested in clinical trials. Those delivered either intranasally or intramuscularly with potent adjuvants were immunogenic and showed positive signs of protection based on human challenge models $(9,10)$. Published studies on the intranasal GI.1 monovalent vaccine (found to be protective in a human challenge model) elicited ASC counts of 50-200 (28), which is the same order of magnitude seen in the present study (ASC counts of 300-500). GMFR IgA antibody responses to one dose of the intranasal monovalent GI.1 VLP increased by approximately 4-fold, which is similar to the increase observed with a single dose of this rAd oral vaccine. In the intramuscular bivalent VLP vaccine, a 5.8 GMFR increase in memory IgA and a 19.1 increase in the memory IgG were reported by Ramani et al. in the highest dose group tested (29). Our study demonstrated skewing toward mucosally primed IgA B cell memory with a GMFR increase of 15 in antigen-specific memory IgA and a 6-fold increase in memory IgG. There have been no reports of direct fecal IgA induction following either intranasal or intramuscular administration of a VLP-based vaccine. However, in studies run by Tacket et al. with an oral VLP vaccine, 3 of 10 subjects had a 4 -fold increase in fecal $\operatorname{IgA}$, with a 3 -fold average increase in the specific IgA response (30). The rAd oral vaccine described herein induced a much stronger IgA response, with a 17- to 36-fold average increase in the same time period.

Noroviruses are a diverse group, with over 30 different genotypes and 6 defined genogroups. Because the number of genotypes represented by a single vaccine is limited, it would be challenging to elicit a host response capable of blocking all norovirus strains from binding target epithelial cells. Inclusion of more than two GLP products in the same vaccine preparation might be expensive and increase the risk of interference. An oral adenoviral-based approach offers the potential advantage of incorporating multiple $V P$ genes in the same GMP vector, and multiple vaccines could be given easily in separate tablets. Another benefit of oral delivery is that the ensuing local IgA responses might be less restricted than those for IgG, allowing for a wider range of functional antibodies to be effective. IgA-mediated protection in the gut relies on immune exclusion in the intestinal lumen and virus excretion from lamina propria in a neutralizing epitope-dependent and -independent manner $(31,32)$. In essence, a cross-strain antibody response may be generated where antibodies incapable of blocking receptor binding directly can still inhibit norovirus infection.

Protection from an enteric pathogen, particularly one like norovirus with rapid induction of severe clinical symptoms, likely relies on fast and local adaptive immune responses at the site of infection. Indeed, studies have shown that mucosal immune responses are as important as serum-blocking antibodies for protection against norovirus gastroenteritis $(9,13-15)$. We hypothesized that oral immunization, where the site of immune activation in the intestine matches the site of norovirus infection, could be the optimal method for generating protective immunity. Ideally, an effective enteric vaccine is expected to elicit memory B cells capable of migrating to the intestinal tissues, which would allow for rapid responses upon infection. Specifically, antigen-specific memory B cells in the vaccinated groups (and expansion of the VP1-specific IgA in stool) were elicited after oral $\mathrm{rAd}$ vaccine administration. In a detailed flow analysis, the vaccine also 
elicited activated memory and effector IgA-committed B cells bearing the $\alpha 4 \beta 7$ homing receptors, indicating their capacity to track to the intestinal mucosa. For polio, which is also an enteric virus, the presence of IgA ASCs expressing $\alpha 4 \beta 7$ markers following oral vaccination has been shown to correlate with protection against shedding following challenge (18). These hypotheses will be tested in future challenge and/or efficacy studies of the rAd oral norovirus vaccine.

In summary, while this was a phase I study and limited in numbers, the single-dose oral rAd-based norovirus vaccine was found to be well tolerated and immunogenic in humans. The broad mucosal immune response induced suggests that this vaccine may offer protection beyond current approaches. Subsequent studies will evaluate multidose and multistrain formats along with vaccine efficacy.

\section{Methods}

Clinical protocol and enrollment. This phase I study evaluated safety and immunogenicity of a rAd5-based oral vaccine against the norovirus GI.1 (Norwalk) strain using 2 dose levels administered at a single clinical site. Enrollment criteria can be found in the Supplemental Methods. The active phase of the trial was through day 28 , with the safety follow-up to monitor for AEs of special interest (AESIs) continuing for 1 year after vaccination.

Randomization and masking. The study was designed to evaluate the vaccine (VXA-G1.1-NN) in 23 subjects at a single low dose of $1 \times 10^{10}$ infectious units (IU) or in 23 subjects at a single high dose of $1 \times 10^{11} \mathrm{IU}$; additionally, 20 subjects were randomized to the placebo control. Initially, 3 sentinel vaccine-treated subjects were sequentially enrolled and assigned to the lower dose level, with each subject dosed no more frequently than once every 24 hours. After 1 week of monitoring for vaccine-related toxicities, 30 subjects were randomized in a 2:1 ratio to either the lower dose vaccine $(n=20)$ or the placebo control $(n=10)$ in a blinded manner. Once the lower dose cohorts were enrolled, the high-dose phase was completed in the same manner. Randomization was performed by computer-generated assignment, and study drug was distributed with concealed identity to the blinded site staff by the unblinded pharmacist. All investigative site staff as well as persons directly involved with immunological assays or the assessment of clinical safety remained blinded to treatment assignments. All subjects were blinded to treatment as well.

Sample size. This phase I trial is a first-in-human study with the VXA-G1.1-NN vaccine candidate. In the absence of clinical data, sample size was determined based on similar phase I vaccine studies and the number predicted to yield meaningful immunogenicity results. A sample size of 20 in each vaccine group and 20 in the placebo group was calculated to provide approximately $86 \%$ power to detect a group difference, assuming the proportion of response (observed in serum IgG VP1) in the vaccine group was $50 \%$ and in the placebo group was $0 \%$, using 2-group Fisher's exact 2-sided test at a significance level of 0.05 (based on commercially available software, nQuery version 8 , by Statistical Solutions Ltd).

Norovirus vaccine. The nonreplicating rAd vector carries DNA, which encodes VP1 from the GI.1 Norwalk strain of norovirus, with expression driven by a CMV promoter and expressed from the missing E1 region of rAd. The vector also encodes a molecular dsRNA hairpin adjuvant driven by a separate promoter. Details of the rAd vector platform have been described previously (27). GMP drug substance was produced in HyClone Single Use Bioreactors (GE Healthcare Life Sciences) at Lonza Biologicals. Purification was performed by ion exchange chromatography, followed by buffer exchange. Purified vector was mixed with excipients, lyophilized, and then tableted at Lonza using microcrystalline cellulose (FMC) and starch (Colorcon) as tableting bulk. Tablets were enteric coated with Eudragit L100 (Evonik Industries) using a Vector Hi-Coater system (Vector Freund). The final product was released in one lot and titered by standard IU assay at Lonza. Placebo was prepared as similarly sized and shaped tablets containing $250 \mathrm{mg}$ microcrystalline cellulose.

Safety assessments. TEAEs were collected from time of initial vaccination. The PI assessed solicited and unsolicited AEs in a blinded manner. The Safety Monitoring Committee oversaw the safety of the study but did not participate in grading AEs. Solicited AEs (reactogenicity) were collected with the aid of a 7-day solicited symptoms diary card. Unsolicited AEs (all other clinical AEs) were collected with the aid of an unsolicited diary card through day 28. To grade AEs, the PI followed the Center for Biologics Evaluation and Research and ref. 33.

Because we believe the adjuvant component of the vaccine to be novel, occurrences of AESI and new onset of chronic illness (NOCI) were also collected. They included neuroinflammatory disorders, musculoskeletal disorders, gastrointestinal disorders, metabolic diseases, skin disorders, and other autoimmune disorders. No AESIs or NOCIs were reported through the end of the monitoring period (365 days after immunization).

Endpoints. The primary endpoint for this study was safety. The secondary endpoint, immunogenicity, was assessed through the active phase (day 28), primarily by BT50 titers to GI.1 VLP. Additional immu- 
nological endpoints investigated included ASCs, specific serum antibody titers, measurement of mucosal homing markers on B cells, fecal and saliva IgA ELISA responses, and memory B cell responses.

BT50 assays. The HBGA-blocking assays (BT50s) have been used historically as a surrogate for neutralizing antibody responses in norovirus studies. These assays measure the ability to block interaction of a blood antigen with a norovirus VLP. The BT50s were qualified and assays were performed by Q2 Solutions, using the method described by Reeck et al. (13). Briefly, biotinylated Le ${ }^{b}$ or H1 (Glycotech) at $2.5 \mu \mathrm{g} / \mathrm{ml}$ was used to coat NeutrAvidin-coated plates (Thermo Fisher Scientific). Two-fold dilutions of serum with GI.1 VLP were incubated at $37^{\circ} \mathrm{C}$ for 1 hour. The mixture of VLP and serum samples was added to HBGA-coated plates and incubated at $4^{\circ} \mathrm{C}$ for 2 hours. After washing, rabbit anti-GI.1 VLP polyclonal antisera (made by Thermo Fisher Scientific specifically for Vaxart) was added and incubated at $4^{\circ} \mathrm{C}$ for 1 hour. Goat anti-rabbit $\operatorname{IgG}(\mathrm{H}+\mathrm{L})$ HRP (Bethyl Laboratories, A120-101P) was used to detect bound rabbit antibody ( $4^{\circ} \mathrm{C}$ for 60 minutes). With the starting dilution of 1:25, a negative value was set equal to 12.5 .

PBMC isolation and cryopreservation. Blood was collected in $\mathrm{K}_{2}$ EDTA Vacutainer tubes from BD, and PBMCs were isolated the same day using Lymphoprep tubes (Axis-Shield). PBMCs were frozen and thawed using serum-free reagents according to the manufacturer's instructions (Cellular Technology Limited [CTL]).

ASCs and ELISA serum antibodies. ASCs were measured using cryopreserved PBMC and enzyme linked immunosorbent (ELISpot) kits for IgG- and IgA-secreting B cells according to manufacturer's instructions (Mabtech). Cells were cultured in triplicate with anti-IgG- or IgA-coated wells in CTL test medium overnight. VP1 VLP from GI.1 made on HEK293 cells (AscentGene) was biotinylated and quantitated using a biotinylation kit (EZ-Link Sulfo-NHS-LC-Biotin kit; Thermo Scientific) and BCA kit (Pierce). Spots were developed using biotinylated VP1 VLP and streptavidin-HRP (Mabtech) and counted at ZellNet Consulting Inc. A positive ASC response was defined as a count after vaccination of at least 3 SDs above the mean prevaccination count and at least 13 spots/well/106 PBMCs (IgG) and 23 spots/well/106 PBMCs (IgA).

IgG and IgA serum antibody responses were measured by ELISA using MaxiSorp plates (Thermo Fisher Scientific) coated with GI.1 VLP $(1 \mu \mathrm{g} / \mathrm{ml})$. Goat anti-human IgG-HRP (MilliporeSigma, AP113P) or human IgA-ALP (Mabtech, 3310-3) was used as a detection antibody. OD versus dilution curves were plotted, and changes in titer at the $\mathrm{EC}_{50}$ were calculated using Prism software (Prism 7).

Flow cytometry. Immunophenotyping was performed on cryopreserved PBMCs as described previously (16). Data were collected on a BD LSR II cytometer using FACS DIVA software and analyzed using FlowJo software (version 10.2). The antibodies to human surface markers were acquired from 4 sources: (a) Biolegend (CD19, clone HIB19; IgG, clone G18-145; CD38, clone HB-7; CCR9, clone L053E8); (b) Miltenyi Biotec (CD27, clone MT271); (c) Dako (IgA, clone F0188); and (d) Thermo Fisher Scientific (37, clone FIB504).

Memory assay. PBMCs in CTL test medium containing $1 \mu \mathrm{g} / \mathrm{ml} \mathrm{R} 848$ (Mabtech) and $10 \mathrm{ng} / \mathrm{ml} \mathrm{recom}$ binant human IL-2 (Mabtech) were incubated for 3 days in U-bottom 96 -well plates $\left(5 \times 10^{5}\right.$ cells per well) at $37^{\circ} \mathrm{C}, 5 \% \mathrm{CO}_{2}$. Cells were harvested and an ASC ELISpot assay performed as described above (19).

Total and VP1-specific stool IgA ELISA. Collection details can be found in the Supplemental Methods. For total IgA determinations, Immulon II microtiter plates (Thermo Fisher Scientific) were coated with purified goat anti-human IgA ( $\alpha$-chain specific) from Jackson Immunoresearch (109-035-011) at $1 \mu \mathrm{g} / \mathrm{ml}$ in PBS for 3 hours at $37^{\circ} \mathrm{C}$. To measure norovirus-specific antibodies, Immulon II plates were coated with G1.1 VLP at 1 $\mu \mathrm{g} / \mathrm{ml}$ in PBS for 3 hours at $37^{\circ} \mathrm{C}$. After incubation, plates were washed with PBS containing $0.05 \%$ Tween 20 (PBST) and blocked overnight at $4^{\circ} \mathrm{C}$ with PBS containing $10 \%$ nonfat dry milk (Nestle). Stool supernatant samples were added to the plates and serially diluted in PBST containing $10 \%$ nonfat dry milk, starting at 1:5,000 for total IgA or at 1:2 for norovirus-specific IgA measurements. After washing in PBST, bound antibodies were detected by incubating plates for 1 hour at $37^{\circ} \mathrm{C}$ with HRP-labeled goat anti-human $\operatorname{IgA}$ (Fc-specific IgA) from Jackson Immunoresearch (109-035-008). Following incubation and washing, TMB Microwell Peroxidase Substrate (KPL Inc.) was added, and plates were incubated for 15 minutes in the dark (with agitation) at ambient temperature. The colorimetric reaction was stopped by the addition of $1 \mathrm{M}$ phosphoric acid to all wells. Absorbance values at $450 \mathrm{~nm}$ were read using a Multiskan FCTM Microplate Reader (Thermo Fisher Scientific). Total and norovirus-specific IgA concentrations were calculated by interpolation of the regression-corrected absorbance values produced by serially diluted samples into a standard curve of purified human IgA (Calbiochem). Results were reported as a ratio of norovirus-specific IgA to total IgA.

Total and VP1-specific saliva IgA. Total IgA saliva antibody concentrations were measured by ELISA. 96-well plates (MaxiSorp, Thermo Fisher Scientific) were coated with capture antibody (MT57, Mabtech) and subsequently blocked with $1 \%$ BSA. Samples were diluted serially from 1:200, and the 
human IgA standard (Mabtech) was serially diluted from $200 \mathrm{ng} / \mathrm{ml}$ before being added to the plates. The bound antibodies were detected by the HRP-conjugated IgA antibody (MT20-ALP, Mabtech) followed by p-nitrophenyl-phosphate substrate. OD values at $405 \mathrm{~nm}$ were measured using a microplate reader (Spectramax M2, Molecular Devices). For specific IgA, MaxiSorp plates were coated with G1.1 VLP $(1 \mu \mathrm{g} / \mathrm{ml})$, and samples were diluted serially starting at 1:2. Total and norovirus-specific IgA concentrations were calculated by interpolation of sample OD values into the standard IgA curve. Results were reported as ratios of VP1-specific IgA to total IgA.

Statistics. Per protocol, an analysis of covariance model was performed on (log-transformed) BT50 titers, and results showed the treated groups to be statistically different from placebo. However, the analysis required normally distributed data, a condition which was found afterward not to have been met. Therefore, nonparametric methods were used for analysis of (log-transformed) neutralizing antibody titers. A Kruskal-Wallis test was used to test for any BT50 titer differences (as well as some additional comparisons between groups) and, if significant, paired-wise vaccine dose group comparisons with placebo were performed using a Mann-Whitney $U$ test. For the additional endpoints, treatment group differences were compared using 2-group Mann-Whitney or unpaired 2-tailed $t$ test ( 2 sided) in continuous variables and Fisher's exact test ( 2 tailed) in categorical variables. $P$ values less than 0.05 were considered significant.

Study approval. The study was conducted in accordance with applicable Good Clinical Practice guidelines, the United States Code of Federal Regulations, and the International Conference on Harmonization guidelines. IRB approval was obtained from the Chesapeake IRB (Columbia, Maryland, USA; AAHRPP accredited) before study-specific screening and enrollment of subjects. Informed consent was obtained from all subjects after discussion of the study procedures and potential risks.

\section{Author contributions}

LK and SNT wrote the paper. KK and KL ran immunological assays. LK, DL, KG, and SNT analyzed the data. DL and SJG designed the clinical protocol. MFP was responsible for the fecal IgA analysis. GT developed the tableting procedure. SJG and DL were responsible for clinical operations.

\section{Acknowledgments}

We would like to thank the study volunteers, the staff of Celerion, the staff at Q2 Solutions, and the staff at Lonza Biologicals for helping us complete this clinical study. Thanks to Hong Farling and Josefina Martinez for performing clinical oversight and providing operational support. We would also like to thank the Vaxart employees who put substantial effort into the undertaking of this study. The authors also wish to thank the Safety Monitoring Committee: Peter Patriarca, Robert Belshe, and John Treanor. We would also like to thank Peter Patriarca for reviewing the clinical protocol and for regulatory advice. Thanks to Elizabeth Li for conducted statistical analyses for this paper. Thanks also to Harry Greenberg, Nikita Kolhatkar, Debora Tingley, and Anne Moore for reviewing the manuscript.

Address correspondence to: Sean N. Tucker, Vaxart, 290 Utah Street \#200, South San Francisco, California 94080, USA. Phone: 650.550.3502; Email: stucker@vaxart.com.

1. Bresee JS, Widdowson MA, Monroe SS, Glass RI. Foodborne viral gastroenteritis: challenges and opportunities. Clin Infect Dis. 2002;35 (6):748-753.

2. Debbink K, Lindesmith LC, Baric RS. The state of norovirus vaccines. Clin Infect Dis. 2014;58(12):1746-1752.

3. Glass RI, Parashar UD, Estes MK. Norovirus gastroenteritis. N Engl J Med. 2009;361(18):1776-1785.

4. Weber DJ, Rutala WA, Miller MB, Huslage K, Sickbert-Bennett E. Role of hospital surfaces in the transmission of emerging health care-associated pathogens: norovirus, Clostridium difficile, and Acinetobacter species. Am J Infect Control. 2010;38 (5 Suppl 1):S25-S33

5. Mitchell C, Meredith P, Richardson M, Greengross P, Smith GB. Reducing the number and impact of outbreaks of nosocomial viral gastroenteritis: time-series analysis of a multidimensional quality improvement initiative. BMJ Qual Saf. 2016;25 (6):466-474.

6. Fankem SL, Boone SA, Gaither M, Gerba CP. Outbreak of norovirus illness in a college summer camp: impact of cleaning on occurrence of norovirus on fomites. J Environ Health. 2014;76 (8):20-26.

7. Bert F, et al. Norovirus outbreaks on commercial cruise ships: a systematic review and new targets for the public health agenda. Food Environ Virol. 2014;6 (2):67-74.

8. Riddle MS, Walker RI. Status of vaccine research and development for norovirus. Vaccine. 2016;34 (26):2895-2899.

9. Atmar RL, et al. Norovirus vaccine against experimental human Norwalk Virus illness. N Engl J Med. $2011 ; 365$ (23):2178-2187.

10. Bernstein DI, et al. Norovirus vaccine against experimental human GII.4 virus illness: a challenge study in healthy adults. $J$ Infect Dis. 2015;211(6):870-878. 
11. Johnson PC, Mathewson JJ, DuPont HL, Greenberg HB. Multiple-challenge study of host susceptibility to Norwalk gastroenteritis in US adults. J Infect Dis. 1990;161 (1):18-21.

12. Kilhamn J, Jertborn M, Svennerholm AM. Kinetics of local and systemic immune responses to an oral cholera vaccine given alone or together with acetylcysteine. Clin Diagn Lab Immunol. 1998;5(2):247-250.

13. Reeck A, et al. Serological correlate of protection against norovirus-induced gastroenteritis. J Infect Dis. 2010;202 (8):1212-1218

14. Lindesmith L, et al. Human susceptibility and resistance to Norwalk virus infection. Nat Med. 2003;9 (5):548-553

15. Ramani S, Estes MK, Atmar RL. Correlates of protection against norovirus infection and disease-where are we now, where do we go? PLoS Pathog. 2016;12 (4):e1005334.

16. Kim L, et al. Systemic and mucosal immune responses following oral adenoviral delivery of influenza vaccine to the human intestine by radio controlled capsule. Sci Rep. 2016;6:37295

17. Liebowitz D, Lindbloom JD, Brandl JR, Garg SJ, Tucker SN. High titre neutralising antibodies to influenza after oral tablet immunisation: a phase 1, randomised, placebo-controlled trial. Lancet Infect Dis. 2015;15 (9):1041-1048.

18. Dey A, et al. Human circulating antibody-producing B cell as a predictive measure of mucosal immunity to poliovirus. PLoS One. 2016;11 (1):e0146010.

19. Jahnmatz M, Kesa G, Netterlid E, Buisman AM, Thorstensson R, Ahlborg N. Optimization of a human IgG B-cell ELISpot assay for the analysis of vaccine-induced B-cell responses. J Immunol Methods. 2013;391 (1-2):50-59.

20. Crotty S, Aubert RD, Glidewell J, Ahmed R. Tracking human antigen-specific memory B cells: a sensitive and generalized ELISPOT system. J Immunol Methods. 2004;286 (1-2):111-122.

21. Streeter PR, Berg EL, Rouse BT, Bargatze RF, Butcher EC. A tissue-specific endothelial cell molecule involved in lymphocyte homing. Nature. 1988;331 (6151):41-46.

22. He XS, et al. Plasmablast-derived polyclonal antibody response after influenza vaccination. J Immunol Methods. 2011;365 (12):67-75.

23. Macpherson AJ, McCoy KD, Johansen FE, Brandtzaeg P. The immune geography of IgA induction and function. Mucosal Immunol. 2008;1 (1):11-22.

24. Mora JR, von Andrian UH. Differentiation and homing of IgA-secreting cells. Mucosal Immunol. 2008;1 (2):96-109

25. Bartsch SM, Lopman BA, Ozawa S, Hall AJ, Lee BY. Global economic burden of norovirus gastroenteritis. PLoS ONE. 2016;11 (4):e0151219.

26. Yuan L, Geyer A, Saif LJ. Short-term immunoglobulin A B-cell memory resides in intestinal lymphoid tissues but not in bone marrow of gnotobiotic pigs inoculated with Wa human rotavirus. Immunology. 2001;103 (2):188-198.

27. Scallan CD, Tingley DW, Lindbloom JD, Toomey JS, Tucker SN. An adenovirus-based vaccine with a double-stranded RNA adjuvant protects mice and ferrets against H5N1 avian influenza in oral delivery models. Clin Vaccine Immunol. 2013;20(1):85-94.

28. El-Kamary SS, et al. Adjuvanted intranasal Norwalk virus-like particle vaccine elicits antibodies and antibody-secreting cells that express homing receptors for mucosal and peripheral lymphoid tissues. J Infect Dis. 2010;202 (11):1649-1658.

29. Ramani S, et al. B-cell responses to intramuscular administration of a bivalent virus-like particle human norovirus vaccine. Clin Vaccine Immunol. 2017;24(5); $00571-16$.

30. Tacket CO, Sztein MB, Losonsky GA, Wasserman SS, Estes MK. Humoral, mucosal, and cellular immune responses to oral Norwalk virus-like particles in volunteers. Clin Immunol. 2003;108 (3):241-247.

31. Mantis NJ, Forbes SJ. Secretory IgA: arresting microbial pathogens at epithelial borders. Immunol Invest. $2010 ; 39$ (4-5):383-406.

32. Mantis NJ, Rol N, Corthésy B. Secretory IgA's complex roles in immunity and mucosal homeostasis in the gut. Mucosal Immunol. 2011;4(6):603-611.

33. Guidance for Industry: Toxicity Grading Scale for Healthy Adult and Adolescent Volunteers Enrolled in Preventive Vaccine Clinical Trials. FDA. https://www.fda.gov/downloads/BiologicsBloodVaccines/ucm091977. Accessed June 15, 2018. 